\begin{tabular}{|c|c|}
\hline $.595(\mathrm{NT})$ & Zhang, S. \\
\hline & Zhang, Z-X....... \\
\hline $\mathbf{Z}$ & 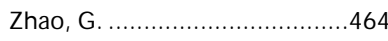 \\
\hline Zamvil, S.S...................1098(NV) & Zhao, Q.H................................... \\
\hline Zanelli, E.............................. & 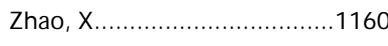 \\
\hline Zeng, W. ............................................ & Zhao, X-Y..................703, 939(Err) \\
\hline Zhang, D. .................................... & Zheng, T.S. .................................. \\
\hline Zhang, L. .............82, 736(NV), 782 & Zhong, R. ...................................... \\
\hline Zhang, M. ..................................... & Zhou, H. ......................... \\
\hline
\end{tabular}

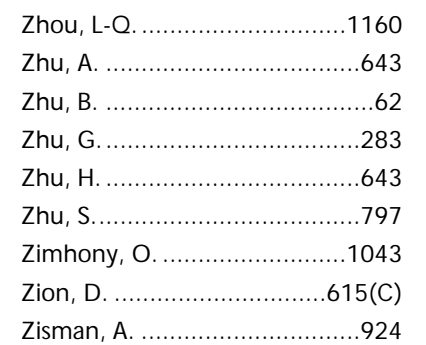

Zlokovic, B.V........................718(L)

Zobywalski, A...........................332

Zöller, G. ...................................32

Zou, Y......................................189

Zuckier, L.S. ................................ 871

Zuscik, M.J............................1388

\title{
2000 Subject index • Volume 6
}

\section{medice

$\begin{array}{lrlc}\text { No. 1 } & 1-111 & \text { No. 7 } & 717-838 \\ \text { No. 2 } & 113-229 & \text { No. 8 } & 839-939 \\ \text { No. 3 } & 231-356 & \text { No. 9 } & 941-1062 \\ \text { No. 4 } & 357-480 & \text { No. 10 } & 1063-1191 \\ \text { No. 5 } & 481-603 & \text { No. 11 } & 1193-1293 \\ \text { No. 6 } & 605-715 & \text { No. 12 } & 1295-1429\end{array}$ \\ (L) Letter to the Editor \\ (N) News \\ (B) Book Review \\ (C) Commentary \\ (NV) News \& Views \\ (NT) New Technology \\ (Corr) Correction \\ (Err) Errata}

\section{A}

Abbott Laboratories, countersuit against, 948(N)

Academic medical centers, industry support group, 948(N)

Achromatopsia, genetic mutation, 746(NV)

Acid sphingomyelinase gene disruption, oocyte apoptosis suppression, 1100 (NV), 1109

Activin A, and basic fibroblast growth factor neuroprotective effect, 739(NV),812

Acute promyelocytic leukemia, transcriptional defects, 742(NV)

Adeno-associated virus vector viral DNA heterodimerization, 599(NT) see also Recombinant adeno-associated virus vector

Adenovirus E1A, and Ewing tumors, 4(L)

Adenovirus mutant dl1520 replication, p14 ${ }^{\text {APF }}$ mechanism, 1128

Adenovirus mutant d1922-947, anti-tumoral efficacy, 1134

Adipogenesis inhibition, AP-1 transcription factor, 970(NV), 985

Adult hippocampal progenitor cells, retinal dystrophy grafts, 1211 (NV)

Africa

HIV vaccine trials, 844(N)

UN acknowledges HIV/AIDS threat, 117(N)

Aging, DNA microarray analysis, fibroblasts, 507(NV)

AIDS, African epidemic, UN acknowledges threat, $117(\mathrm{~N})$

AIDS vaccine, 1220 (NV). See also HIV vaccine

Akt activation, statin mechanism of action, 965(NV), 1004

Albinism, gene therapy approach, mice, 27(NV)

Alcohol intake, fetal neurotoxicity mechanisms, 263(NV)

Allogeneic bone marrow transplantation. See Bone marrow transplantation
Allografts. See Transplantation

Alpha-hemolysin, urinary tract pathogenesis, 741(NV)

Altered-peptide ligand, multiple sclerosis clinical trials, 1098(NV), 1167,1176

Alternative medicine, 240(N)

Alzheimer disease

amyloid $\beta$-peptide antibody treatment, mice, 916

amyloid $\beta$-peptide improper clearance,

133(NV), 143

ibuprofen treatment

magnetic resonance microscopy, 20(NV)

American Heart Association, stem cell research support, 845(N)

$\delta$-aminolevulinate dehydratase import mechanism, 898

AMPA antagonists, multiple sclerosis potential use, 15(NV), 62,67

Amygdala, morphine sensitization, SPARC glycoprotein, 910

Amyloid-A-induced RAGE upregulation, 633(NV),643

Amyloid $\beta$-peptide

antibody treatment, mouse model, 916

catabolic pathway identification,

133(NV), 143,718(L)

microvascular endothelium transport, 718(L) pathological deposition mechanism,

133(NV), 143

Amyloid $\beta$-protein precursor

cytotoxic proteolytic peptide derivation, caspase cleavage, 385(NV),397

neurodegeneration direct role, 512(NV)

Amyloid plaques, magnetic resonance microscopy, 20(NV)

Amyloidosis

amyloid-A-induced RAGE upregulation, 633(NV),643 and yeast prions, 751(R)

Anderson, Roy, 1070(N)

Androgen escape mechanism, prostate cancer, 628(NV), 703,933(Err)

Anergy, p2 $7^{\mathrm{kip} 1}$ function, 290

Ang1, dermal vessels effect of, 131(NV)

Angiogenesis

Ang1 and VEGF effect, 131(NV)

ischemic peripheral neuropathy therapy, VEGF gene transfer, 379(NV), 405

maspin inhibition of, 196

mechanisms and therapeutic application,

389(R)

PR39 regulation of, 49,356(Err)

thrombospondin-1 inhibition of, signals, 41

tumor and wound healing mechanism,

979(NV)

vascular endothelial growth factor gene

therapy risk, 1102(NV)

xenogeneic endothelial cell anti-tumor

immunotherapy, 1160

Angioma-genesis, VEGF gene therapy risk, 1102(NV)

Angiopoietin-1, adult vasculature leakage protection, 460

Ank mouse arthritis model, genetic defect elucidated

Anthrax, Russian outbreak investigation, 245(B)

Antibiotics

deterrence of resistance to, 142(NV)

VncS-VncR pathway target, 263(NV)

Antigen-specific regulatory $T$ cells, 782

Antigen-specific T cells, in situ detection, 1056(NT)

Anti-infective biomaterials, screening, 1053(NT)

Anti-inflammatory effects, carbon monoxide, 422

Anti-retroviral therapy. See Highly active anti-retroviral therapy

Anti-tumor therapy. See Cancer therapy AP-1 transcription factors, bone formation, 
970(NV),980,985

Apoptosis

mitochondrial control, 513(R)

thrombospondin-1 induction of, signals, 41

TRAIL induction of, hepatocytes, 502(NV),564

Aquaporin-4 deletion, cerebral edema reduction, 159

Arginine-cyclosporin A conjugation, topical delivery, 1253

Arntzen, Charles, 364(N)

Arteriogenesis, mechanisms, 389(R)

Atherosclerosis, herpesvirus role, 979(N)

Attention deficit/hyperactivity disorder, basal ganglia functional deficits, MRl, 470

Australia

biomedical lobbying group created, $613(\mathrm{~N})$

Celera Genomics Corporation deal, 847(N)

Ogilvie's research funding warning, 725(N)

scientists' discontent, survey, 10(N)

universities told to commercialize, $120(\mathrm{~N})$

Australian Aborigines, group A streptococcal vaccine, 455

Autoimmune disease

CD40 agonistic antibodies, arthritis,

629(NV), 673

gram-negative infection link, molecular

mimicry model, 215

human cytomegalovirus link, 1183

and thymocyte positive selection disruption, 259(NV), 298

Autoimmune T cells, multiple sclerosis, 15(NV),56

B-cell inhibition, limitin role, 659

B-cell malignancies, T-cell response induction, 667

B-cell mitogen identification, pathogenic trypanosome, 865(NV),890

Barbacid, Mariano, 239(N)

Basal ganglia functional deficits, attention deficit/hyperactivity disorder, 470

Basic fibroblast growth factor neuroprotection, and activin A induction, 739(NV), 812

Baylor University, science and religion center controversy, 613(N)

BBB scale scores, 358(L)

Bcl-2 oncoprotein, chemoresistance mechanism, 1029

Beanie Baby profits, AIDS Foundation support, 726(N)

$\beta$-cell induction, PDX-1 role, hepatocytes, 505(NV), 568

$\beta$-human chorionic gonadotropin, in tumor assessment, 711(NT)

Bioengineering, FY00 congressional priority, 7(N)

Bioethics, 615(C)

Bio-flavonoids, infant leukemias role, 512(NV)

Bioinformatics, and functional genomics, 1071(N)

Biomaterials, anti-microbial screening, 1053(NT)

Biomedical education, 'Supercourse' proposal, 358(L)

Biomedical publication, ethics, 1205(B)

Biomedical research survey shows secrecy in, 365(N)

trends, 113(E)

Blood stem cells, renal cell carcinoma treatment, 1107(NV)

Blower, Sally, 359(N)

Bone cancer pain, osteoprotegerin treatment, 504(NV),521,838(Err)

Bone formation,

AP-1 transcription factor family in, 970(NV), 980,985

statin stimulation of, $21(\mathrm{NV})$

Bone marrow transplantation

and co-stimulatory blockade, macrochimerism and tolerance induction, 464

graft-versus-host disease prevention, 18(NV)

"facilitating cells" surface marker, 866(NV), 904

Books reviewed

Anthrax: The Investigation of a Deadly

Outbreak, 245

Cancer: The Evolutionary Legacy, 496

Darwin's Ghost: The Origin of Species Updated 621

Defending the Cavewoman, 371

The Door in the Dream: Conversations With

Eminent Women in Science, 1089

Driving Mr. Albert: A Trip Across America with

Einstein's Brain, 1090

Ethical Issues in Biomedical Publication, 1205

A Fever in Salem; A N ew Interpretation of the

New England Witch Trials, 127

Flu: The Story of the Great Influenza Pandemic of 1918 and the Search for the Virus that

Caused It, 12

Genome: The Autobiography of a Species in 23

Chapters, 11

The M ethuselah Gene: A Science Fiction

Adventure Thriller, 857

Neural Transplantation, 621

Of Mice, Men, and Microbes-Hantavirus, 11

The Paradox of Sleep, 245

The Shape of the Heart, 857

Stress, Coping, and Depression, 733

To America's Health: A Proposal to Reform the

Food and Drug Administration, 963

The Triple Helix: Gene, Organism and

Environment, 1206

Virus: The Co-Discoverer of HIV Tracks Its

Rampage and Charts the Future, 372

Xeno: The Promise of Transplanting Animal

Organs into Humans, 963

Borst, Piet, 8(N)

Bovine spongiform encephalopathy, 258(NV)

Brain edema, aquaporin-4 modulating role, 159

Brain tumors

magnetic resonance spectroscopy, nosologic images, 1287(NT)

neural stem cells, gene therapy vehicle,

369(C), 447

Brazil, research centers long-term support, 1198(N)

BRCA test, NIH pays cut-rate prices, $610(\mathrm{~N})$

Breast cancer iodide transporter expression, 859(NV),871 selective estrogen receptor modulators, 960(C)

Bullous impetigo, exfoliative toxin A mechanism, 1213(NV), 1275

C

Calcineurin, and heart failure, 2(L)

Calcium signaling, cardiac growth/function, $1221(R)$

Canada

health research legislation, $8(\mathrm{~N})$

networked research expansion, 240(N)

Cancer

clinical trial insurance coverage agreement,

1212(N)

evolutionary legacy, 496(B)

metastasis model, 100

transcription regulation

Cancer research

UK NCI proposal, 357(E)

UK parliamentary committee inquiry, 360(N)

UK report suggests improvements, $945(\mathrm{~N})$

Cancer therapy

adenovirus mutant dl1520 replication facilita-

tion, 1128

adenovirus mutant d1922-947, 1134

cannabinoid agonists, 255(NV),313

Fc receptors cytotoxicity role, 373(NV), 4434

gene therapy clinical trial, 862(NV),879

MGATS inhibitors potential, 306

vesicular stomatitis virus, 821

Cancer vaccine

renal carcinoma, 252(NV), 332

TERT RNA transfected dendritic cells, 966(NV),1011

xenogeneic endothelial cells, 1160

Cannabinoids, anti-tumor action, 255(NV),313

Carbon monoxide, anti-inflammatory effects, mechanism, 422

Carcinoembryonic antigen, positron emission tomography, 869(NV)

Cardiac failure. See Heart failure

Cardiac growth/function, calcium signaling, $1221(\mathrm{R})$

Cardiac hypertrophy calcium signaling, 1221(R) myocyte adaptation, myosin light chain kinase, 183

TAK1 role, transgenic mice, 556 transgenic mice models, need for controls, 482(L)

Cardiomyocytes

HF-1 $1 \beta$ null mice, $968(\mathrm{NV})$

myosin light chain kinase role, hypertrophy, 183

Cardiovascular disease, bacterial infections role, 841(L)

Carlsson, Arvid, 1196(N)

Carney complex gene discovery, 979(NV)

Caspase activation, compensatory pathways, 1241

Caspase-cleaved amyloid $\beta$-protein precursor, cyto- 
toxic proteolytic peptide generation, 385(NV),397

Caspase 8 suppression, neuroblastoma M YCN amplification, 498(NV),529

CCR5 co-receptor, intercellular transfer, 769

$\mathrm{CD} 4$ co-receptor function, video microscopic analysis, 1097(NV)

$\mathrm{CD}^{+}$bone marrow transplant facilitating cell, surface marker, 866(NV), 904

$\mathrm{CD} 8^{+}$cells

HIV homeostasis impact, 976(NV),1036 virus- and tumor-specificity detection, 1056(NT)

$\mathrm{CD}^{+}{ }^{+}$cells, flow cytometry counting, 833(NT)

CD36, thrombospondin-1 antiangiogenic activity mediation, 41

CD40 agonistic antibodies, in chronic autoimmune inflammatory disease, 629(NV), 673

CD40 ligand blockade of, thromboembolic complications, 114(L) dendritic cell expression, $\mathrm{CD}^{+}$cellindependent immunity, 1154

CD45 mutations, severe combined immunodeficiency disease, 343

Celera Genomics sequence data, financial optimism, scientific skepticism, 116(N)

Celiac disease, dominant A-gliadin T-cell epitope identification, 337

Cell death, mitochondrial control, 513(R)

Cell physiology, 1087(C)

Cell signaling 'glue grant,' 1072(N)

Cellular transformation, transcriptional regulation, 742(NV)

'Central DNA flap,' 626(NV)

Ceramide accumulation, cannabinoid apoptosis mechanism, 255(NV), 313

Ceramide analogs, infertility protection potential, 1100(NV), 1109

Cerebral edema, aquaporin-4 modulating role, 159

Chagas disease

NSAIDs potential use in, 142(NV) proline racemase as B-cell mitogen, 865(NV), 890

Challenge Grants program, 1197(N)

Chemokines, T-cell repulsive response, 543

Chemoresistance analysis, murine lymphoma model, 1029

Chemotherapy

dosage design, 500(NV)

ONYX-015 combination, clinical trial, 862(NV), 879

Chimpanzees, US sanctuary system, 9(N)

China, first international HIV vaccine meeting, 5(N)

Cholesterol homeostasis, retinoid $\mathrm{X}$ receptor role, 1104(NV)

Cholesteryl ester transfer protein inhibitor, 869(NV)

Chronic fatigue syndrome, GAO funding controversy report, 846(N)

Chronic myelogenous leukemia, T-cell immunity in elimination of, 1018

Cis-activation strategy, recombinant adeno- associated virus vector, 595(NT)

Clinical research

industry support, 948(N)

NIH progress report, 1201 (C)

Clinical scientists, UK plans, 490(N)

Clinical trials research, standards, 1193(E)

ClinicalTrials.gov, 365(N)

Coeliac disease. See Celiac disease

Collagen-induced arthritis, CD40 agonist antibody treatment, 629(NV), 673

Colonic fluid production, galanin-1 receptor up-regulation, 1048

Colony-stimulating factor, placental immunity role, 589

Colorectal cancer, combinatorial chemoprevention, 874(NV), 1024

Combinatorial chemoprevention, intestinal neoplasia, 974(NV), 1024

Combined co-stimulatory blockade failure, transplant model, 115(L)

Congestive heart failure. See Heart failure

Cortisol-responsive androgen receptor mutants, prostate cancer, 628(NV), 703

Coxsackievirus-mediated heart disease tyrosine kinase $\mathrm{p} 56^{\text {lck }}$ role, 429 viral damage versus molecular mimicry model, 631(NV), 693

Creutzfeldt-Jakob disease, 'new variant,' 258(NV)

Crohn disease, interleukin 6 trans signaling role, 583

CTLA-4 blockade, T-cell paradoxical inhibition, heterogeneity, 211

Cyclooxygenase signaling inhibition, combinatorial chemoprevention, 974(NV), 1024

Cyclopentenone prostaglandins, anti-inflammatory mechanism, 137(NV)

Cyclosporin A-arginine conjugation, topical delivery, 1253

Cysteine protease calpain 10 gene, Type II diabetes, 1107

Cytokines

heat-shock protein-70 production of, 429 transplantation rejection regulation, 497(NV), 549

Cytomegalovirus infection, autoimmunity link, 1183

Cytotoxic T cells, leukemia elimination, 1018

\section{D}

Darwin's theory, 621(B)

DDT, balance of risk paradigm, 729(C)

Declaration of Helsinki, 615(C)

$\Delta$-aminolevulinate dehydratase import mechanism, 898

Demyelinating diseases, endogenous sodium channel blocker role, 738(NV), 808

Dendritic cells

CD40L expression, and CD4 cell-independent immunity, 1154

Dengue virus target, 748(NV), 816

scrapie pathogenesis role, 719(L)

TERT RNA transfection, tumor immunity,

966(NV), 1011 tumor cell hybrid vaccine, renal carcinoma, 252(NV), 332

Dengue virus, dendritic cells target of, 748(NV), 816

Dentate gyrus, neuronal progenitor cell isolation, 249(NV), 271

Depression, and stress, 733(B)

Desmoglein 1, exfoliative toxin A target, 1213(NV), 1275

Diabetes mellitus See Type I diabetes; Type II diabetes

Diarrhea, infectious, galanin-1 receptor upregulation, 1048

Dickman, Michael, 121(N)

Diltiazem, rod photoreceptor rescue, 116(L)

Disability Adjusted Life Expectancy, 722(N)

Disgust, specialized brain system, 1220(NV)

d1922-947, anti-tumoral efficacy, 1134

dl1520 adenovirus mutant, replication facilitation, 1128

DNA microarray analysis, fibroblast aging mechanism, 507(NV)

DNA mismatch repair. See Mismatch repair

DNA vaccine

fetal oral cavity administration, 929

measles, 744(NV), 776, 1062(Err)

Doxorubicin-peptide 'prodrug,' 1248

Dreaming, 245(B)

Drosophila, nucleotide sequence revealed, 387(NV)

Drug addiction, neurotrophic factors role, 635(NV)

Drug resistance analysis, murine lymphoma model, 1029

Duchenne muscular dystrophy, gene therapy, golden retrievers, 6353(NV)

Dysentery, 'probiotic' treatment, 257(NV), 265

E

Ebola virus glycoprotein, pathogenicity determinant, 886

Einstein's brain, 1090(B)

Embryonic stem cells. See Human embryonic stem cells

Endothelial xenogeneic cells, antitumor immunotherapy, 1160

Environmental endocrine hypothesis, 246(B)

Epidermal growth factor inhibition, combinatorial chemoprevention, 974(NV), 1024

Epidermal powder immunization, 1187(NT)

Erythrocytes, ring stage $P$. falciparum infection, 1264

Escherichia coli infection $\alpha$-hemolysin urinary tract pathogenesis, 741(NV)

'probiotic' treatment, 257(NV), 265

Ethics. See Research ethics

Ewing tumors, and adenovirus E1A, 4(L)

Exfoliative toxin A, epidermal blistering mechanism, 1213(NV), 1275

Experimental autoimmune encephalomyelitis AMPA antagonists in, 15(NV), 62, 67 glutamate receptor blockade, 15(NV), 62, 67 thymic autoreactive $\mathrm{T}$-cell repertoire role, 
15(NV), 56

Experimental colitis, interleukin 6 trans signaling, 583

Expressed-sequence tags patents, revisions, 362(N)

$\mathbf{F}$

Facilitating cell surface marker, bone marrow transplantation, 866(NV), 904

Familial Alzheimer disease, presenilin mutations, 1220(NV)

Familial hemophagocytic lymphohistiocytosis, 27(NV)

Fas-associated death domain protein, inflammatory gene expression activation, 790

Fas engagement, liver regeneration acceleration, 920

FasL, immune privilege mechanisms re-evaluation, 493(C)

Fat transplantation, and diabetes studies, 263(NV)

Fatty acid synthetase I, pyrazinamide inhibition, 977(NV), 1043

Fatty liver disease reversal, metaformin effect, mice, 998

Favaloro, Rene, 1070(N)

Fc receptors, anti-tumor cytotoxicity modulation, 373(NV), 443

$\mathrm{Fc} \alpha \mathrm{RI}$, serum IgA interactions, mucosal immunity, 680

Feeding behavior, glucagon-like peptide-2 role, 802

Fetal alcohol syndrome, mechanisms, 263(NV)

Fetal hemoglobin, sickle cell disease, transgenic mice, 139(NV), 177

Fetal immunization, DNA vaccine oral cavity delivery, 929

Fetal loss, coagulation factors gene mutations, 1220 (N)

Fetal stem cells. See Human embryonic stem cells

Fibroblasts, aging mechanism, DNA microarray analysis, 507(NV)

Flow cytometry counting, CD34+ cells, 833(NT)

FOG-2, heart development role, 864(NV)

Folkman, Judah, 948(N)

Follicular dendritic cells, scrapie pathogenesis, 719(L)

Follicular epithelial cells, as skin epidermis source, 1095(NV)

Food and Drug Administration, proposal to reform, 963(B)

Food intake, glucagon-like peptide-2 role, 802

Ford, Larry C., 614(N)

Fra-1 overexpression, bone formation, 970(NV),980

Framingham Heart Study, commercialization, 721(N)

Functional antibodies, design of, 123(C)

Functional genomics, bioinformatics, 1071(N)

Functional MRl, attention deficit/hyperactivity disorder, 470

Fundacion Favaloro, 1070(N)

\section{G}

Galanin-1 receptor up-regulation, excess colonic fluid production, 1048
Gamma interferon, vascular xenogeneic rejection role, 497(NV), 549

Gankyrin overexpression, retinoblastoma protein destabilization, hepatomas, 96

Gastric atrophy attenuation, concurrent helminth and helicobacter infection, 536

Gastric cancer, Helicobacter pylori link, 376(NV)

Gastritis, concurrent helminth and helicobacter infection, 536

Gender differences, HIV-1 genetic diversity, 23(NV), 71

Gene expression

in vivo magnetic resonance imaging, 256(NV), 351

positron emission tomography, 933(NV)

Gene patents, revisions, 362(N)

Gene therapy

annual meeting reinvigoration of field, $722(\mathrm{~N})$

cancer Phase II clinical trial, 862(NV), 879

cautious optimism, 717(E)

clinical trial monitoring changes, $486(\mathrm{~N})$

neural stem cells as vehicle, brain tumors,

369(C), 447

Parkinson disease, 1207(NV)

severe combined immunodeficiency, 623(NV)

sickle cell disease, mouse model, 139(NV),

177

Gene therapy death, 6(N)

biotechnology industry response, $118(\mathrm{~N})$

clinical trial monitoring changes, $486(\mathrm{~N})$

safety wake-up call, 1(E)

Senate subcommittee hearing, 235(N)

Gene transfer, hematopoietic stem cells, 624(NV), 652

Genetic databases, United Kingdom plan, 359(N)

Genetic testing, insurance premium assessment use, UK, 1199(N)

Genetically modified organisms, UK research difficulties, 364(N)

Germ line protection, and cancer therapy, 1100(NV), 1109

Glial cell line-derived neurotrophic factor, Parkinson disease gene therapy, 1207(NV)

Glioma therapy

cannabinoid agonists, 255(NV), 313

chimeric poliovirus, 750(NV)

neural stem cells, IL-4 delivery, 369(C), 447

Global Alliance for Vaccines and Immunizations commercial boost, 363(N)

fund raising, $238(\mathrm{~N})$

Glucagon-like peptide-2, food intake regulation role, 802

Glucocorticoid-responsive androgen receptor mutants, prostate cancer, 628(NV), 703, 939(Err)

Glucose, transdermal monitoring using ultrasound, 347

Glucose intolerance, glucose transporter 4 importance, 924

Glucose transporter 4 targeted disruption, 924

'Glue grant' announced, 1072(N)

Glutamate receptors blockade of, multiple sclerosis model, 15(NV) 62,67

immunization against, neuroprotective effects, 383(NV)

Government-University Research Partnership, 239(N)

Graft-versus-host disease donor cell manipulation, 18(NV)

and LIGHT pathway, 283

Grafts. See Transplantation

Gram-negative pathogens, autoimmune disease link, 215

Green fluorescence protein neural progenitor cell expression, 271, 483(L) transgenic expression of, cardiomyopathy cause, 482(L)

Greengard, Paul, 1196(N)

Grinstein, Sergio, 485(N), 609(N)

Group A streptococcal vaccine, Australian Aborigines, 455

Guillain-Barré syndrome, endogenous sodium channel blocker role, 738(NV), 808

Gulf War syndrome, magnetic resonance spectroscopy, 750(NV)

H

HAART. See Highly active anti-retroviral therapy Hair follicle epithelial cells, skin epidermis source, 1095(NV)

Hamilton, W.D., 367(Obit)

Hantavirus, 11(B)

Hcrt mutation, early onset narcolepsy, 991

Head and neck cancer, gene therapy clinical trial, 862(NV), 879

Health Life Expectancy Rankings, 722(N)

Heart disease

bacterial infections role, $841(\mathrm{~L})$

calcium signaling, $1221(\mathrm{C})$

Heart failure

and calcineurin, 2(L)

gene therapy, 512(NV)

sarcoplasmic reticulum function, 942(L)

TAK1 role, transgenic mice, 556

Heart function, calcium signaling, 1221(R)

Heat-shock protein-70, chaperone and cytokine dual role, 429

Helicobacter pylor

concurrent helminth infection, gastric atrophy attenuation, 526

gastic cancer link, 376(NV)

Helminth infection, gastric atrophy attenuation, 536 Hemangioma risk, vascular endothelial growth factor gene therapy, 1102(NV)

Hematopoietic stem cells engraftment facilitation, 18(NV) hepatic regeneration, 1212(NV), 1229 long-term gene transfer, 624(NV), 652 Notch signaling immortalization of

1210(NV), 1278

p2 $7^{\mathrm{kip} 1}$ effects, 1235

Hepatectomy, Fas-induced apoptosis prevention, 920 
Hepatic regeneration, hematopoietic stem cells, 1212(NV), 1229

Hepatitis B infection, hepatocyte xenotransplantation model, 327

Hepatitis C virus, 1082(C)

Hepatitis $\mathrm{C}$ virus infection long-term immunological correlates of recovery, 578

outcome prediction, 512(NV) vaccine advance, mouse model, 142(NV)

Hepatitis delta virus infection, hepatocyte xenotransplantation model, 327

Hepatocarcinogenesis, gankyrin overexpression, 96 Hepatocyte transplantation human hepatitis infections model, 327 variable therapeutic effect, mice, 320

Hepatocytes

hematopoietic stem cell contribution, 1212(NV), 1229

NF- $\kappa B$ immune response function, 573

PDX-1 induced conversion to $\beta$-cells, 505(NV), 568

TRAIL-induced apoptosis, 502(NV), 564

transplantation, 320, 327

HER-2/neu, PEA3 suppression of, tumorigenesis inhibition, 189

Herpesvirus, atherosclerosis role, 979(NV)

Heyward, William, 361(N)

HF-1b disruption, ventricular arrhythmias, 968(NV)

Highly active anti-retroviral therapy

episomal HIV-1 replication persistence, 76

HIV-1 primary infection, viremia control,

1094(NV), 1140

and latent reservoir HIV-1 replication, 76, 82,

736(NV), 757

non-latent reservoir viral rebound, 736(NV), 757

Hippocampus, neuronal progenitor cell isolation, 249(NV), 271, 483(L)

HIV discovery, 372(B)

HIV-1 evolution, 750(NV)

HIV-1 genetics, gender differences, 23(NV),71

HIV-1 infection

CCR5 co-receptor mechanism, 769

mycophenolic acid control of, 735(NV), 762

naïve T-cell homeostasis impact, 976(NV),

1036

nuclear translocation mechanism, 626(NV)

oral polio vaccine theory, 1067(NV)

HIV-1 nuclear translocation mechanism, 626(NV)

HIV-1 pathogenesis, gender differences, 23(NV), 71

HIV-1 protease mutations, online search engine for queries, 1290(NT)

HIV-1 rebound, HAART discontinuation, 76, 82, 736(NV), 757

HIV-1 replication

episome-specific PCR detection, HAART, 76

HAART limitations, 76, 82

latent reservoirs of, 76,82

mycophenolic acid control of, 735(NV), 762

HIV-1 reverse transcriptase mutations, online search engine for queries, 1290(NT)
HIV-1/SIV chimeric virus, mucosal transmission protection, 129(NV), 200, 207

HIV-1 specific CD4 ${ }^{+}$cells homeostasis, 976(NV), 1036 latent virus source, 736(NV), 757

HIV-1 therapy, 261(NV). See also Highly active anti-retroviral therapy

HIV-1 transmission and HIV-1 genetic diversity, 23(NV), 71 in vitro models, mucosa, 475(NT), 607(L) mother-to-infant, mucosal IgG protection, 129(NV), 200

mucosal protection, IgGs, 129(NV), 200, 207 organ culture model, female genital tract, 475(NT), 607(L)

HIV-1 vaccine

African trials planned, $844(\mathrm{~N})$

China's international meeting, $5(\mathrm{~N})$

design and construction of, Kenya trial,

951(C)

IgG mucosal protection, SHIV chimeric virus,

129(NV), 200, 207

Kenya clinical trial, 951(C)

$\mathrm{NIH}$ revamps clinical trials unit, 488(N)

QUEST collaborative trial, 1194(L)

South African trial, 1199(N), 1200(N)

UNAIDS guidelines, 363(N)

US NIAID funding, 844(N)

HLA types, South African ethnic groups, 3(L)

HRAS oncogene, Kaposi sarcoma-associated herpesvirus oncogenesis mechanism, 1091(NV), 1121

HTLV-1, South American origin, 232(L)

Human cytomegalovirus, virion mRNA content, 863(NV)

Human cytomegalovirus late protein VL94, autoimmunity link, 1183

Human genome, 11(B)

Human embryonic stem cells, 231(E) activists threaten academic research funding, 119(N)

adult stem cells effect of, 747(NV)

American Heart Association support, 845(N)

Europe asks UK to reject cloning approval, 1068(N)

European bioethical discussion, 845(N)

Japanese guidelines, 239

$\mathrm{NIH}$ guidelines delay, 231(E)

Pope influences scientists, 1069(N)

UK approves research, 950(N)

UK policy speculation, 486(N)

University of Wisconsin sale of, 237(N)

US guidelines released, 1068(N)

US Senate subcommittee debate, $614(\mathrm{~N})$

Huntingtin

expression block, 512(NV)

Hereditary Disease Foundation conference, 1063(E)

normal and mutant function, $1208(\mathrm{NV})$

Huntington disease

Hereditary Disease Foundation conference, 1063(E) huntingtin expression block, 512(NV)

minocycline mortality delay, mice, 797

normal and mutant huntingtin role

Hyaluronic acid, Plasmodium falciparum adhesion, placenta, 25(NV), 86

Hypocretins, narcolepsy role, 991

Hypoxia-inducible factor-1 $\beta$, PR39 inhibition of, 49

Ibuprofen, Alzheimer's disease treatment, 973(NV)

IgG, mucosal HIV transmission protection, 129(NV), 200, 207

IкB kinase, cyclopentenone prostaglandins inhibition of, 137(NV)

Immune Tolerance Network, "Edmonton Protocol" commences, 950(N)

Immunocompetent SCID-hu mice, 103(NT)

Immunofluorescence, gamma interferon and II-10 detection, 107(NT)

Immunoglobulin framework-derived peptides, cytotoxic T-cell epitope function of, 667

Immunoglobulin A, FcoRl interactions, mucosal immunity, 680

Immunologic 'ignorance,' vascularized organ transplants, 686

Imperial College School of Medicine, 1070

Infectious diarrhea, galanin-1 receptor upregulation, 1048

Infertility

ceramide analogs protective effects, mice, 1100(NV), 1109

spermatogonial cell transplantation, mice, 16(NV), 29

Inflammatory disease, carbon monoxide therapeutic potential, 422

Inflammatory response, signaling systems link, 137(NV)

Influenza pandemic of 1918, 12(B)

Influenza vaccine, needle-free powder delivery, 1187(NT)

Informed consent, 615(C)

Innate immune system, trophoblast pregnancyspecific component, 589, 838(Corr)

Institutional Review Boards, 1193(E) Insulin-dependent diabetes. See Type I diabetes Insulin resistance

glucose transporter 4 importance, 924 metaformin therapeutic mechanism, mice, 998

skeletal muscle respiratory uncoupling prevention strategy, 1092(NV), 1115

Insurance premium assessment, genetic testing use, UK, 1199(N)

Interferon- $\gamma$ coxsackievirus-induced myocarditis protection, 631(NV),693 high-sensitivity immunofluorescence detection, 107(NT)

Interleukin-2, p2 $7^{\mathrm{kip} 1}$ inhibition of, 290

Interleukin-4, neural stem cell delivery, gliomas, $369 \odot, 447$

Interleukin-6 trans signaling, intestinal inflammation 
pathogenic pathway, 583

Interleukin-10,high-sensitivity immunofluorescence detection, 107(NT)

Interleukin-12, transplantation regulation, 497(NV), 549

International Financial Institution Advisory Commission HIV report, 491(N)

Intestinal inflammation, interleukin-6 trans signaling role, 583

Intestinal neoplasia, combinatorial chemoprevention, 974(NV), 1024

Intrauterine growth retardation, thromboxane $\mathrm{A}_{2}$ receptor role, 219

Intravascular metastasis model, 100

lodide transporter expression, mammary tumors, 859(NV), 871

Iron transporter, 263(NV)

Ischemic peripheral neuropathy, VEGF gene transfer therapeutic angiogenesis, 379(NV), 405

Islet cell transplantation, 750(NV)

Isocitrate lyase, tuberculosis latent stage infection role, 979(NV)

Israel, medical research quality decline, $9(\mathrm{~N})$ Italy

biomedical research budget, $9(\mathrm{~N})$

genomics project, 1071(N)

neuroscience network plans, 490(N)

J

Japan

human embryonic stem cell research

guidelines, 239(N)

Institute of Medical Science career structure changes, $488(\mathrm{~N})$

Juvenile Diabetes Foundation, research funding increase, $726(\mathrm{~N})$

\section{K}

Kandel, Eric, 1196(N)

Kaposi sarcoma-associated herpesvirus latent nuclear antigen oncogenesis mechanism, 1091(NV), 1121 reservoir in great apes, 1107(NV)

Kay, Marguerite, 120(N)

Kenya, HIV-1 vaccine clinical trial, 951(C)

Kety, Seymour S., 727(Obit)

Koren, Gideon, 364(N), 485(N), 609(N)

Kourilsky, Philippe, 236(N)

Kringle 5 lawsuit, 723(N)

Krumlauf, Robert, 949(N)

Ku80 function, 503(NV)

Lamins, 136(NV)

Langerhans cells, dengue virus target, 748(NV), 816

Large multifunctional proteasome-2, in non-obese diabetic mouse, 1064(L)

Lasker Awards, 1073(C), 1082(C), 10878(C)

Leishmaniasis, miltefosine treatment, 27(NV)

Leptin, diabetes role, 263(NV)

Leukemia cell death, superoxide dismutase inhibition, 1105(NV)
Life expectancy rankings, 722(N)

LIGHT, immunoregulatory functions, mice, 283

Limitin, lymphopoiesis inhibition, 659

Lipid-tagged antibody fragments, as functional cell-surface receptors, 233(NT)

Liver disease, hepatocyte transplantation variable therapeutic effect, 320

Liver regeneration bone marrow cells role, 869(NV) Fas engagement, 920

Liver-stage antigen 3, malaria immunization, 1218(NV), 1258

LM NA mutations, 136(NV)

Locomotor sensitization, SPARC glycoprotein role, 910

Lupus erythematosus. See Systemic lupus erythematosus

Lustgarten Foundation, 605(E)

\section{M}

Macrophage migration inhibitory factor, sepsis role, 140(NV), 164

Magnetic resonance imaging attention deficit/hyperactivity disorder, 470 in vivo transgene expression, 256(NV), 351

Magnetic resonance microscopy, amyloid plaques, 20(NV)

Magnetic resonance spectroscopy nosologic images, brain tumors, 1287 (NT)

Magnetofluorescent liposomes, 107(NT)

Malaria, 941(E)

challenges and initiatives, $941(\mathrm{E})$

drug target identification, 898

European vaccine research, 234(L)

funding increase, Africa, $612(\mathrm{~N})$

immunity target identification, 689

immunization with liver-stage antigen 3 ,

1218(NV), 1258

$M S P-1_{19}$ vaccine antigen, functional

conservation, 91

Nature M edicine Special Focus website, 720(N)

P. falciparum hyaluronic acid adhesion,

placenta, 25(NV), 86

transmission-blocking vaccine development,

241(C)

vaccine obstacles, 955(C)

Male infertility, spermatogonial cell transplantation, mice, $16(\mathrm{NV}), 29$

Malignant glioma therapy, cannabinoid agonists, 255(NV), 313

Mammary gland, iodide transporter expression, 859(NV), 871

Marijuana, opposition to new research rules, 10(N)

Maspin

angiogenesis inhibition, 196

tumor suppression mechanism, 374(NV)

Massachusetts Institute of Technology, neuroscience center, 362(N)

Mast cell tryptase, neurogenic inflammation mechanism, PAR2 agonism, 134(NV), 151

Mbeki, Thabo, 843(N)

MCP Hahnemann University, censure escape,
724(N)

Measles, DNA vaccine, 744(NV), 776, 1062(Err)

Medical Research Council, research staff neglect accusation, 725(N)

The Medical Scientist Training Program, 605(E)

Medulloblastoma, REST/NRSF regulator, 826, 1062(Err)

Mendoza, Carmen, 609(N)

Meningococcal pathogenesis, functional genomics, 1215(NV), 1269

Mental health research, funding distribution questioned, 237(N)

Mesenchymal stem cells, xenogeneic transplantation, site-specific differentiation, 1282

Messenger RNA 3' untranslated region, pathology 'hotspot?' 637(R)

Metastatic tumors. See Tumor metastasis

Metformin, fatty liver disease reversal, 998

Mgat5-deficient mice, tumor suppression, 306

MHC class Ib molecules, autoimmune disease link, 215

MHC-peptide multimers, T cell detection and sorting, 707(NT)

Michael Polanyi Center, 613(N)

Mifepristone, “off label” research, 1196(N)

Miltefosine, leishmaniasis treatment, 27(NV)

Minocycline, Huntington disease mortality delay, mice, 797

Mismatch repair, MLH3 identification, 19(NV)

MIT neuroscience center, 362(N)

Mitochondria, cell death control, 513(R)

Mitogen-activated protein kinase pathway, carbon monoxide anti-inflammatory effects mechanism, 422

Molecular mimicry coxsackievirus-induced myocarditis, 631(NV),693 infection and autoimmune disease model, 215

Molecular population genetics, malaria immunity target identification, 689

Monoclonal antibodies, antitumor therapy, Fc receptors, 373(NV), 443

Montagnier center fate, 235(N)

Morphine sensitization, SPARC glycoprotein role, amygdala, 910

MSP- $1_{19}$, functional conservation across Plasmodium species, 91

Mucosal HIV transmission, in vitro models, 475(NT), 607(L)

Mucosal immunity, serum IgA and Fc $\alpha$ RI interactions, 680

Mucosal SHIV neutralizing antibodies, 129(NV), 200, 207

Multidrug resistance 1 gene, long-term transfer, 624(NV), 652

Multiple sclerosis altered peptide ligand clinical trials, 1098(NV), 1167, 1176 AMPA antagonists in, 15(NV), 62, 67 endogenous sodium channel blocker role, 738(NV), 808 
glutamate receptor blockade, 15(NV), 62, 67 multiple approaches, 15(NV), 56, 62, 67 thymic autoreactive $\mathrm{T}$-cell repertoire role, 15(NV), 56

Murine lymphoma model, chemoresistance analysis, 1029

Muscle differentiation, calcium regulation mechanism, 1216(NV)

Muscle wasting, NF-kB role, 1216(NV)

Muscular dystrophy, gene therapy, golden retrievers, 635(NV)

MYCN amplification, caspase 8 suppression role, neuroblastomas, 498(NV), 529

Mycobacterium tuberculosis 'cording' mechanism, 632 (NV) pyrazinamide mechanism, drug design implications, 977(NV), 1043

Mycophenolic acid, HIV-1 replication control, 735(NV), 762

Myelin basic protein ${ }_{83-99}$, MS clinical trials, 1098(NV), 1167, 1176

Myocardial hypertrophy, TAK1 role, transgenic mice, 556

Myocarditis, coxsackievirus mechanism, 429, 631(NV), 693

Myogenesis mechanism, 1216(NV)

Myosin light chain kinase, sarcomere organization mediation, 183

\section{N}

Narcolepsy

Hcrt mutation, early onset case, 991 hypocretin peptides, 991

National Cancer Institute, UK readiness question, 357(E), 360(N)

National Institutes of Health Challenge Grants, program, 1197(N) clinical research progress report, 1201(C) gender biology research funding, 950(N) graduate student training programs expansion, 847(N)

HIV vaccine unit restructurization, 488(N) pharmacogenetics initiative, $487(\mathrm{~N})$ 2001 budget plans announcement, 239(N)

Natural killer cells, tumor cell recognition, 867(NV)

$N$ eisseria meningitidis pathogenesis, functional genomics, 1215(NV), 1269

Neovascularization thrombospondin-1 inhibition of, signals, 41 See also Angiogenesis

Neural plasticity, and premature infant pain memories, 971(NV)

Neural stem cells development potential, 747(NV) gene therapy vehicle, brain tumors, 369(C), 447 safety, 369(C)

Neural transplantation, 621(B), 634(NV)

Neuroblastomas, caspase 8 suppression with M YCN amplification, 498(NV), 529

Neurogenic inflammation, proteinase-activated receptor 2 agonists, 134(NV), 151
Neuronal progenitor cells

enhanced green fluorescent protein effect on 483(L)

in vitro neurogenesis, 249(NV), 271, 483(L)

retinal dystrophy grafting, rat model, 1211(NV)

Neuronal repressor REST/NRSF, medulloblastoma regulator, 826

Neuroprotective autoimmunity, glutamate receptors, 383(NV)

Neuroscience research center, MIT gift, 362(N)

NF-KB

hepatocyte immune function role, 573

muscle wasting role, 1216 (NV)

NKG2D receptor, tumor cell killing activation, 867(NV)

NMDA receptor, immunization against, neuroprotective effects, 383(NV)

Nobel Prize in Physiology or Medicine, 1196(N)

Non-obese diabetic mouse, proteasome activity, 1064(L)

Non-steroidal anti-inflammatory drugs, Alzheimer disease treatment, 973(NV)

Nosological images, magnetic resonance spectroscopy, brain tumors, 1287 (NT)

Notch signaling, hematopoietic stem cell immortalization, 1210(NV), 1278

Novartis (Cambridge, UK), BioTransplant(Boston, US) merger, 1195(N)

NREM sleep, neuronal control of, 510(NV)

Nuclear envelope, lamins interaction, 136(NV)

\section{0}

Obesity prevention, respiratory uncoupling prevention strategy, 1092(NV), 1115

Office for Human Research Protection investigations, 946(N)

Oka vaccine strain, frequent reactivation evidence, 381(NV), 451

Olfactory ensheathing glia, spinal cord repair, 382(NV)

Oligonucleotide arrays. See DNA microarray analysis Olivieri, Nancy, 364(N), 485(N), 609(N)

ONYX-015

chemotherapy combination, clinical trial, 862(NV), 879

replication facilitation, 1128

Oral cavity DNA vaccine delivery, fetal lambs, 929

Oral polio vaccine-AIDS theory, contradictory evidence, 1067(N)

Oral squamous cell carcinomas, genetics, $141(\mathrm{NV})$

Orexin, in enteric neurons, 130(NV)

Organ culture model, HIV-1 transmission, 475(NT)

Organ transplantation. See Transplantation

Orphan drugs, NIH Challenge Grants program, 1197(N)

'Orphan receptors', cholesterol homeostasis role, 1104(NV)

Osteoclasts, paramyxovirus upregulation of, 387(NV)

Osteoporosis, statins potential use, 21(NV)

Osteoprotegerin, bone cancer pain treatment,
504(NV) ,521, 838(Err)

Osteosclerosis, AP-1 transcription factors role, 970(NV), 980, 985

Otog function, 142(NV)

\section{P}

P14 ${ }^{\mathrm{APF}}$ loss, adenovirus mutant dl1520 replication, 1128

p21, sex-linked lupus development role, 171

p27 $7^{\mathrm{kp} 1}$, anergy role, 290

p53

and adenovirus mutant dl1520 use, 1128

maspin gene regulation, $374(\mathrm{NV})$

p56 ${ }^{\text {lck }}$, coxsackievirus B3-mediated heart disease role, 429

Paget disease, paramyxoviruses role, 387(NV)

Pain memories, premature infants, 971(NV)

Pancreatic stem cells, insulin-dependent diabetes reversal, 250(NV), 278

Paradoxical sleep, 245(B)

Parainfluenza virus type-1, RhoA as antiviral target, 35

Parathyroid hormone, thymus as secondary source of, 860(NV)

Parkinson disease

glial cell line-derived neurotrophic factor delivery, 1207(NV)

new models, 387(NV)

Pasteur Institute reform, 236(N)

Patient safety, HHS criticizes US research centers, 611(N)

PDX-1 induced insulin gene expression, hepatocytes, 505(NV), 568

PEA3, HER-1/neu suppression, tumorigenesis inhibition, 189

Pediatric Rule, new drug applications, 1069(N)

Pemphigus foliaceus pathogenesis, 869(NV), 1213(NV), 1275

Perforin, familial hemaphagocytic lymphohistiocytosis role, 27(NV)

Peripheral vasoconstriction, plethysmographic detection, REM sleep, 606(L)

Pharmacogenetics

NIH initiative, 487(N)

private-public partnerships need, $48(\mathrm{E})$, 487(N)

Photoreceptor disc defects, and retinal disease, 508(NV)

Photosensitive epilepsy, 263(NV)

Physician scientists concern over numbers of, 605(E) UK plans, 490(N)

Physics, and biomedical research, 113(E)

Phytomedicines, FYOO congressional priority, 7(N)

Pigmentation restoration, gene therapy approach, 27(NV)

Pingelapese color blindness, 746(NV)

Placebo clinical trial ethics, developing countries, 1198(N)

Placental malaria, P. falciparum hyaluronic acid adhesion, 25(NV), 86

Plasmodium berghei, $\delta$-aminolevulinate dehydratase 
as drug target, 898

Plasmodium falciparum

hyaluronic acid adhesion, placenta, 25(NV),

86

immunity target identification, 689

liver-stage antigen 3 protection against,

1218(NV), 1258

ring-stage infected erythrocytes,

cytoadhesion, 1264

Plasmodium species, MSP- $\mathrm{I}_{19}$ functional

conservation, 91

Plethysmography, peripheral vasoconstriction detection, REM sleep, 606(L)

Pluripotent stem cells

insulin-dependent diabetes reversal, 250(NV),

278

See also Human embryonic stem cells

Poly-Q tracts, neurodegeneration role, 27(NV)

Population genetics, malaria immunity target identification, 689

Positron emission tomography

reporter gene expression imaging, 933(NT)

tumor-specific antibody fragmentation,

869(NV)

Post-transfusion hepatitis, 1082(C)

Powerject vaccine delivery, 1187(NT)

PR39, angiogenesis regulation, 49, 356(Err)

Pre-eclampsia, neurokinin $\beta$ link, 750(NV)

Pregnancy, trophoblast innate immune response role, 589, 838(Corr)

Pregnancy complications, coagulation factors gene mutations, 1220(NV)

Premature infants, pain memories, 971(NV)

Presenilin mutations, capacitative calcium entry downregulation, 1220(NV)

Prion protein. See also Transmissible spongiform encephalopathy

amyloidoses link, 751(R)

human enteric nervous system expression,

840(L)

normal function, 1107(NV)

Private-public partnerships, 481(E), 617(C)

Proline racemase, $\mathrm{T}$. cruzi B-cell mitogen, 865(NV), 890

Prostate cancer

androgen escape mechanism, 628(NV), 703,

939(Err)

doxorubicin-peptide 'prodrug' selectivity,

1248

trogliatozine therapeutic potential, 1107(NV)

Prostate-specific antigen, peptide-doxorubicin ‘prodrug' activation, tumor cell killing, 1248

Proteasome activity, non-obese diabetic mouse, 27(NV), 1064(L)

Proteinase-activated receptor 2 agonists, neurogenic inflammation, 134(NV), 151

Public health

HIV health care needs link, 261(NV)

public-private partnerships for, $617(\mathrm{C})$

Public-private partnerships, 481(E), 617(C)

PubMed Central, 496(N)

Pulmonary hypertension reversal, serine elastase in- hibitor, 698

Pyrazinamide, fatty acid synthetase I inhibition, 977(NV), 1043

Q

The Quest trial, 1194(L)

R

Radioiodide, in breast cancer, 859(NV), 871

RAGE regulation

amyloidosis mechanism, 633(NV), 643

cancer suppression, 635(NV)

Reactive oxygen species, cancer therapy target, 1105(NV)

Recombinant adeno-associated virus vector, cis-activation strategy, 595(NT)

REM sleep, peripheral vasoconstriction, plethysmographic detection, 606(L)

Renal cell carcinoma

blood stem cell treatment, 1107(NV)

tumor cell-dendritic cell hybrid vaccine,

252(NV), 332

Renault, Beatrice, $4(\mathrm{~N})$

Reporter gene expression, PET imaging, 933(NT)

Research!America, 613(N)

Research data access, White House OMB rules, 8(N)

Research ethics, 615(C)

Respiratory syncytial virus, RhoA as antiviral target, 35

REST/NRSF, medulloblastoma regulator, 826, 1062(Err)

Retinal disease, photoreceptor disc defect link, 508(NV)

Retinal dystrophy, neural grafts, rat model, 1211(NV)

Retinitis pigmentosa, diltiazem rod photoreceptor rescue, $116(\mathrm{~L})$

Retinoblastoma-E2F pathway, KSHV oncogenesis mechanism, 1091(NV), 1121

Retinoblastoma protein, destabilization by gankyrin overexpression, 96

Retinoid $\mathrm{X}$ receptor heterodimers, cholesterol regulation, 1104

Rheumatoid arthritis, CD40 agonist antibody treatment, 629(NV), 673

RhoA-derived peptide, syncytium formation inhibition, 35

Rhodopsin, and light-induced retinal degeneration, 508(NV)

Rituximab, 373(NV), 443

'River theory,' 489(N)

Rod photoreceptor rescue, diltiazem use, 116(L)

Royal Society 'River theory' discussion,489(N)

\section{$\mathbf{S}$}

S-phase cells, visualization, 387(NV)

Sachs, Jeffrey, 491(N)

Salem witch trials, 127(B)

Salk-UCSD institute? 489(N)

Santos, Eugenio, 238(N)

Sarcomere organization, myosin light chain kinase mediation, hypertrophy, 183
Sarcoplasmic reticulum function, heart failure, 942(L)

Schizophrenia, genetics, 253(NV)

SCID-hu immunocompetent mice, antigen-specific immune response, 103(NT)

Scientific misconduct, 946(N)

Scrapie, follicular dendritic cells role, 719(L)

Secretory leukocyte protease inhibitor, wound healing role, 1147

Selective estrogen receptor modulators, 960(C) Sepsis

macrophage migration inhibitory factor role, 140(NV), 164

superoxide anions role, 979(NV)

Serine elastase inhibitors, pulmonary hypertension reversal 698

Severe combined immunodeficiency disease CD45 mutations, 343 gene therapy, 623(NV)

Sex differences. See Gender differences

Shelby law, 121(N)

Shiga toxigenic infections, 'probiotic' treatment, 257(NV), 265

Sickle cell disease, gene therapy, mouse model, 139(NV), 177

Signature-tagged mutagenesis, N. meningitidis, 1215(NV), 1269

Simian-human immunodeficiency virus IgG mucosal protection, 129(NV), 200, 207 vaccine advance, 1220 (NV)

Simian immunodeficiency virus, primary infection viremia control, 1094(NV), 1140

Simvastatin, angiogenesis promotion, molecular pathway, 965(NV), 1004

Single-chain antibody fragments, lipid-tagging method, 223(NT)

Skin grafts, combined costimulatory blockade failure, 115(L)

Skin stem cells, hair follicular epithelial cells as source of, 1095(NV)

Sleep-promoting neurons, identification of, 510(NV)

Sodium channel blocker, demyelinating disease role, 738(NV), 808

South Africa HIV/AIDS policy inactivity, 843(N)

HIV mixed messages, 1200 (N)

HIV vaccine trial, 1199(N), 1200(N)

HLA types, 3(L)

Soybeans, infant leukemias role, 512(NV)

Space travel-induced bone loss, 635(NV)

Spain

oncology center under threat, $847(\mathrm{~N})$

oncology research centers, 238(N)

Oncology Research Institute's scientists win reprieve, $485(\mathrm{~N})$

'Spanish' influenza pandemic of 1918, 12(B)

SPARC glycoprotein, morphine sensitization mechanism, 910

Spermatogonial transplantation, fertility restoration, mice, 16(NV), 29

Sphingosine-1-phosphate therapy, oocyte apoptosis 
suppression, 1100(NV), 1109

Spinal cord injury

functional recovery definition, 358(L)

human olfactory ensheathing glial cell

transplants, 382(NV)

Split AAV vectors strategy, 599(NT)

Squamous cell carcinoma, gene therapy clinical trial, 862(NV), 879

Staphylococcal scalded-skin syndrome, 1213(NV), 1275

Statins

anabolic effects on bone, 21(NV)

angiogenesis promotion, Akt activation, 965(NV), 1004

Stem cells

developmental potential, 747 (NV)

p27 $7^{\mathrm{kip} 1}$ effects, 1235

somatic support cells regulation of, 1220 (NV)

See also Human embryonic stem cells

Stop TB program, 491(N)

Stowers Institute, 949(N)

Straus, Stephen, 240(N)

Streptococcus pyrogenes

crystal structure features, 387(NV)

toxic shock syndrome protection, 414

Stress, and depression, 733(B)

Stroke, neuroprotective autoimmunity, glutamate receptors, 383(NV)

Stromal cell derived factor-1, T-cell repulsive response to, 543

Sudden cardiac death, HF-1b null mice, 968(NV)

Superantigen, therapeutic peptide antagonist, 378(NV), 414

'Supercourse' proposal, 358(L)

Superoxide dismutase inhibition, cancer therapy, 1105(NV)

Systemic lupus erythematosus

p21 role, model, 171

and thymocyte positive selection disruption, 259(NV), 298

Systemic sclerosis, human cytomegalovirus late protein UL94 link, 1183

$\mathbf{T}$

T cell receptor excision circle, HIV infection, 976(NV), 1036

T cells

activation domain, 378(NV), 414

active repulsive response to chemokine, 543

antigen-specific suppression, 782

B-cell malignancies induction of

immunogenic peptides, 667

CD4 activation, video microscopy, 1097(NV)

chronic myelogenous leukemia elimination,

1018

cytokines effect, graft rejection, 497(NV), 549

detection and sorting, 707(NT)

homeostasis of, HIV impact, 976(NV) ,1036

p21 role in proliferation of, 171

p2 $7^{\mathrm{kp} 1}$ anergy role, 290

paradoxical inhibition, CTLA-4 blockade, 211

tumor cell target recognition, 867(NV) virus- and tumor-specificity detection, 1056(NT)

TAK1, heart failure role, transgenic mice, 556

Tamoxifen, estrogen receptor biology, 960(C)

Telomerase-deficient mouse, 849(C), 852(C)

Telomerase reverse transcriptase RNA transfected dendritic cells, tumor immunity, 966(NV), 1011

Telomere biology, human versus mouse, cancer, 849(C), 852(C)

TERT RNA transfected dendritic cells, tumor immunization, 966(NV), 1011

3' untranslated mRNA region, pathology 'hotspot,' 637(R)

Thromboembolic complications, CD40 ligand blockade, 114(L)

Thrombospondin-1, neovascularization inhibition, signals, 41

Thromboxane $\mathrm{A}_{2}$ receptor, intrauterine growth retardation role, 219

Thymic dysfunction impact, HIV infection, 976(NV), 1036

Thymic T-cell repertoire, and multiple sclerosis, 15(NV), 56

Thymocytes, autoimmune disease mechanism, 259(NV), 298

Thymus, parathyroid hormone source, 860(NV)

Toxic shock syndrome protection, superantigen antagonist, 378(NV), 414

TRAIL, apoptosis induction, normal human hepatocytes, 502(NV), 564

Transcription factors, cellular transformation regulation, 742(NV)

Transfusion-associated hepatitis, 1082(C)

Transgene expression, in vivo magnetic resonance imaging, 256(NV), 351

Transmissible spongiform encephalopathies follicular dendritic cells role yeast prions, 751(R)

Transplantation

allocation squabble, US, 611(N)

combined costimulatory blockade failure,

$115(\mathrm{~L})$

cytokine regulation, 497(NV),549

hepatocyte variable therapeutic effect, 320

HIV patients, 365(N)

nerve cells, 621(B), 634(NV)

neuronal progenitor cells, hippocampus,

249(NV), 271

pancreatic stem cells, 250(NV), 278

and secondary lymphoid tissue absence,

immunologic 'ignorance,' 686

Trastuzumab, 373(NV), 443

Troglitazone, prostate cancer protection, 1107(NV)

Trophoblast, pregnancy-specific innate immune system component, 589, 838(Corr)

Trypanosoma cruzi, Chagas disease mechanism, 865(NV), 890

Trypanosome survival, cell death relationship, 142(NV)

Trypsin, neurogenic inflammation role, PAR2 agonism, 134(NV),151
Tuberculosis

'cording' mechanism, pcaA, 632(NV)

isocitrate lyase role, 979(NV)

pyrazinamide mechanism, drug design impli-

cations, 977(NV), 1043

Stop TB initiative, 491(NV)

Tumor cells, natural killer cell attack mechanism, 867(NV)

Tumor growth suppression, Mgat5 role, 306

Tumor metastasis

chemotherapy dosage design, 500(NV)

Fc receptors cytotoxicity modulation, 373 ,

443

intravascular endothelial origin, model, 100

maspin gene mechanism, 374(NV)

mechanisms, 500(NV)

Mgat5 inhibitors role, 306

Tumor necrosis factor-related apoptosis-inducing ligand. See TRAIL

Tumorigenesis, HER-2/neu overexpression suppression, 189

Type I diabetes

pancreatic stem cell reversal of, 250(NV),278

PDX-1-induced insulin gene expression,

hepatocytes, 505(NV), 568

proteasome defect, 27(NV), 1064(L)

Type II diabetes

CAPN 10 gene association, 1107(NV)

glucose transporter 4 importance, 924

skeletal muscle respiratory uncoupling prevention strategy, 1092(NV),1115

Typhoon Lengkieki, 746(NV)

Tyrosine phosphatase CD45 gene mutations, and SCID, 343

Tyrosinemia type I, hematopoietic stem cell therapy, 1212(NV),1229

\section{U}

Ubiquitin system, 1073(C)

Ulcerative colitis, interferon 6 trans signaling role, 583

Ultrasound, glucose transdermal monitoring, 347

UN peacekeeping troops, AIDS threat, 949(N)

UNAIDS, HIV vaccine guidelines, 363(N)

Uncoupling protein expression, obesity prevention, 1092(NV), 1115

United Nations, HIV/AIDS threat to world peace acknowledged, 117(N)

University of California San Francisco, gender discrimination charge, 359(N)

University of Nebraska Medical Center, fetal stem cell research threatened, 119(N)

\section{V}

Vaccination

global initiative, 238(N)

Welfare-based sanctions program expanded, 119 (N)

Vaccines

commercial boost, 363(N)

dendritic cells-renal carcinoma cells, tumor

regression, 252(NV),332 
functional antibody design, $123(\mathrm{C})$

global initiative, 238(N)

needle-free powder delivery, 11878(NT)

Third World development, industry

perspective, 723(N)

Varicella-zoster Oka vaccine, 381(NV), 451

Vascular endothelial growth factor

angiogenesis mechanism, 131(NV)

angio-genesis or angiogenesis stimulation?

1102(NV)

ischemic peripheral neuropathy therapeutic angiogenesis, 379(NV), 405

Vascular grafts, cytokine regulation, 497(NV), 549

Vascular leakage protection, angiopoietin-1, 460

Vascular smooth muscle cells, Fas/FADD-mediated inflammation, 790

Ventricular cardiomyocytes, HF-1b null mice,
968(NV)

Ventrolateral preoptic hypothalamic region, sleep control, 510(NV)

Veronesi, Unberto, 610(N)

Vesicular stomatitis virus, oncolytic effects, 821

West Nile virus, US resurgence anticipated, 947(N) Women

clinical trials enrollment progress, $612(\mathrm{~N})$

HIV-1 genetic diversity, 23(NV), 71

NIH gender biology research funding, 950(N)

Women in science, 1089(B)

World AIDS Conference, 613(N),839(E)

World Health Organization

HIV drug policy attacked, 726(N)

institutional framework problems, 481(E),

491(N)
Stop TB participation, 491(N)

Wound healing, secretory leukocyte protease inhibitor role, 1147

Wounds, tumor similarity, angiogenesis, 979(NV)

Xenogeneic endothelial cells, antitumor immunotherapy, 1160

Xenotransplantation, 963(B) cytokine regulation of, 497(NV), 549 hepatocytes, hepatitis infection model, 327 Novartis-BioTransplant merger signals research shift, $1195(\mathrm{~N})$ UKXIRA delays potential trial approvals, $10(\mathrm{~N})$

Yeast prions, 751(R) 